\title{
XMMU J050722.1-684758: Discovery of a new Be X-ray binary pulsar likely associated with the supernova remnant MCSNR J0507-6847
}

\author{
C. Maitra, ${ }^{1 \star}$ F. Haberl, ${ }^{1}$ P. Maggi, ${ }^{2}$ P. J. Kavanagh,${ }^{3}$ G. Vasilopoulos, ${ }^{4}$ \\ M. Sasaki ${ }^{5}$ M. D. Filipović, ${ }^{6}$ A. Udalski ${ }^{7}$ \\ ${ }^{1}$ Max-Planck-Institut für extraterrestrische Physik, Gießenbachstraße, 85748 Garching, Germany \\ ${ }^{2}$ Université de Strasbourg, CNRS, Observatoire astronomique de Strasbourg, UMR 7550, F-67000 Strasbourg, France \\ ${ }^{3}$ School of Cosmic Physics, Dublin Institute for Advanced Studies, 31 Fitzwillam Place, Dublin 2, Ireland \\ ${ }^{4}$ Department of Astronomy, Yale University, PO Box 208101, New Haven, CT 06520-8101, USA \\ ${ }^{5}$ Remeis Observatory and ECAP, Universität Erlangen-Nürnberg, Sternwartstr. 7, 96049 Bamberg, Germany \\ ${ }^{6}$ Western Sydney University, Locked Bag 1797, Penrith South DC, NSW 2751, Australia \\ ${ }^{7}$ Astronomical Observatory, University of Warsaw, Aleje Ujazdowskie 4, 00-478 Warsaw, Poland
}

Accepted XXX. Received YYY; in original form ZZZ

\begin{abstract}
We report the discovery of a new high mass X-ray binary pulsar, XMMU J050722.1-684758, possibly associated with the supernova remnant MCSNR J0507-6847 in the Large Magellanic Cloud, using XMM-Newton X-ray observations. Pulsations with a periodicity of $570 \mathrm{~s}$ are discovered from the Be X-ray binary XMMU J050722.1-684758 confirming its nature as a HMXB pulsar. The HMXB is located near the geometric centre of the supernova remnant MCSNR J0507-6847 (0.9' from the centre) which supports the XRB-SNR association. The estimated age of the supernova remnant is $43-63 \mathrm{kyr}$ years which points to a middle aged to old supernova remnant. The large diameter of the supernova remnant combined with the lack of distinctive shell counterparts in optical and radio indicates that the SNR is expanding into the tenous environment of the superbubble N103. The estimated magnetic field strength of the neutron star is $B \gtrsim 10^{14} \mathrm{G}$ assuming a spin equilibrium condition which is expected from the estimated age of the parent remnant and assuming that the measured mass-accretion rate remained constant throughout.
\end{abstract}

Key words: X-rays: individuals: XMMU J050722.1-684758, MCSNR J0507-6847-X-rays: binaries - ISM: supernova remnants - Radio continuum: ISM - Radiation mechanisms: general - Magellanic Clouds

\section{INTRODUCTION}

A neutron star (NS) X-ray binary associated with its parent supernova remnant (SNR) is an extremely rare object and can provide unique insights on the early evolutionary stages of NSs in the presence of a binary companion. The visibility time of a SNR is only a few $10^{4} \mathrm{yr}$, which is typically three orders of magnitude shorter than the lifetime of high-mass X-ray binaries (HMXBs). A HMXB-parent SNR association therefore implies a very young binary system. A majority of these associations have been found in the Magellanic Clouds (MCs) in recent years, given their ideal environment for hosting young stellar remnants, a high formation efficiency for high-mass X-ray binaries (HMXBs), as well as rela-

\footnotetext{
^ E-mail: cmaitra@mpe.mpg.de
}

tively small distance and low foreground absorption conducive to performing detailed studies.

Discovered XRB-SNR associations include LXP 4.4 (Maitra et al. 2019), SXP 1062 (Haberl et al. 2012; González-Galán et al. 2018), CXO J053600.0-673507 (Seward et al. 2012), SXP 1323 (Gvaramadze et al. 2019) in the Magellanic Clouds, and SS 433 and Circinus X-1 in our Galaxy (Geldzahler et al. 1980; Heinz et al. 2013). The youngest among them until now are Circinus X-1, with an estimated age $<4600$ years (Heinz et al. 2013) and LXP 4.4 with an estimated age of $<6000$ years (Maitra et al. 2019).

MCSNR J0507-6847 is a candidate SNR in the LMC (Bozzetto et al. 2017) that was first reported by Chu et al. (2000) as RX J050736-6847.8, a large ring ( $\sim 150 \mathrm{pc})$ of diffuse X-ray emission projected in the vicinity of the superbubble LHA 120 N 103 (hereafter N103). Superbubbles are large structures in the interstellar medium created by the supernova explosions of massive 
stars and their stellar winds in an OB association or stellar cluster. The shock heated gas in superbubbles emit in X-ray wavelengths. Detection of excess of diffuse X-ray emission in superbubbles are indicative of the presence of interior SNRs shocking the inner walls of the superbubble shell (see for e.g. Dunne et al. 2001).

The above category of SNRs expand in the low-density medium of the superbubble, and have very weak optical and radio emission associated with them. Therefore, the nature of these systems cannot be confirmed using the conventional SNR diagnostics i.e. presence of a high $\left[\mathrm{S}_{\mathrm{II}}\right] /[\mathrm{H} \alpha]$ line ratio and non-thermal radio emission coincident in X-ray emission. The candidate MCSNR J0507-6847 was likewise indicated to be the largest SNR in the LMC expanding in the low density environment of the superbubble N103, and hence with no discernible optical emission (Magellanic Clouds Emission Line Survey) and radio continuum emission (Chu et al. 2000; Bozzetto et al. 2017; Yew et al. 2020). The X-ray luminosity of the system lies within the range expected for SNRs, and the age was estimated to be $\sim 5 \times 10^{4} \mathrm{yr}$ based on the Sedov solution (Chu et al. 2000).

In this work we identify for the first time the BeXRB XMMU J050722.1-684758 and MCSNR J0507-6847 as a possible SNR-HMXB association and investigate the properties of the SNR and its compact object in detail. We report the discovery of pulsations from the BeXRB XMMU J050722.1-684758 located near the geometrical centre of the SNR candidate MCSNR J0507-6847. This confirms its nature as an NS. van Jaarsveld et al. (2018) identified the source as a BeXRB in the LMC with the optical companion classified as a B3 IIIe star and suggested a binary orbital period of 5.27 days. We identified a more likely orbital period of 40.2 days using more than 22 years of OGLE monitoring data. The observations and their analysis are described in Section 2. Section 3 presents the results and Section 4 the discussion and Section 5 the conclusions.

\section{OBSERVATIONS AND ANALYSIS}

\subsection{X-ray observations and analysis}

XMMU J050722.1-684758 was observed serendipitously with XMM-Newton twice in 2000 (Obs1 from now) and again in 2017 (Obs2 from now). The observation details are given in Table 1. EPIC (Strüder et al. 2001; Turner et al. 2001) observations were processed with the XMM-Newton data analysis software SAS version 18.0.0 ${ }^{1}$. We searched for periods of high background flaring activity by extracting light curves in the energy range of 7.0-15.0 keV and removed the time intervals with background rates $\geq 8$ and $2.5 \mathrm{cts} \mathrm{ks}^{-1} \operatorname{arcmin}^{-2}$ for EPIC-PN and EPIC-MOS respectively (Sturm et al. 2013). Events were extracted using the SAS task evselect by applying the standard filtering criteria (\#XMMEA_EP \&\& PATTERN $<=4$ for EPIC-PN and \#XMMEA_EM \&\& PATTERN $<=12$ for EPIC-MOS).

The Swift/XRT data were analysed following the Swift data analysis guide ${ }^{2}$ (Evans et al. 2007). Because of the low flux of XMMU J050722.1-684758 in all observations, we only performed a simple source detection and position determination using a slidingcell detection algorithm implemented by XIMAGE and sosta ${ }^{3}$. For

\footnotetext{
1 Science Analysis Software (SAS): http://xmm.esac.esa.int/sas/

2 http://www.swift.ac.uk/analysis/xrt/

3 https://heasarc.gsfc.nasa.gov/xanadu/ximage/ximage. html
}

non-detections we estimated the $3 \sigma$ upper limits using a Bayesian method introduced by Kraft et al. (1991).

\subsection{OGLE}

The infrared counterpart of the HMXB XMMU J050722.1-684758 is identified to be 2MASS 05072214-6847592 with $J, H$ and $K$ magnitudes of $15.0,15.3$ and 14.6 mag respectively. 2MASS 05072214-6847592 was observed by the Optical Gravitational Lensing Experiment (OGLE), which started observations in 1992 (Udalski et al. 1992) and continued observing until today (OGLE-IV, Udalski et al. 2015), but interrupted since March 2020. Optical observations were performed with the $1.3 \mathrm{~m}$ Warsaw telescope at Las Campanas Observatory, Chile. Images are taken in the $\mathrm{V}$ and I filter pass-bands and photometric magnitudes are calibrated to the standard VI system.

\section{RESULTS}

\subsection{Morphology of MCSNR J0507-6847}

Figures 1 and 2 display the combined XMM-Newton EPIC image centered on MCSNR J0507-6847 overlaid with optical (MCELS) and radio (ASKAP) contours. The source morphology resembles a large circular shell-like structure of diffuse emission of $\sim 150 \mathrm{pc}$ in diameter. It is projected in the vicinity of the superbubble N103 adjacent to SNR N103B (MCSNR J0508-6843) in the east (Maggi et al. 2016; Bozzetto et al. 2017). The north-eastern part of the shell overlaps with the hot gas of the superbubble which is also indicated by the presence of strong optical $\mathrm{H} \alpha$ and radio-continuum emission coincident with this region. The south-western shell is more clearly defined. No optical or radio emission is detected from the shell region which is possibly due to the fact that MCSNR J0507-6847 expands in the low-density environment of the superbubble. To measure the size of the shell, we employed the method described by Kavanagh et al. (2015), which fits an ellipse to the outer contours of the shell (at $3 \sigma$ above the surrounding background level in the $0.2-1 \mathrm{keV}$ EPIC image). Due to the contamination of the northern shell region with the SB N103 as seen from the $\mathrm{H} \alpha$ contours in Fig. 2, the extended emission from the north is used as a background component to define the contour around the northern shell. We derive the ellipse centre at R.A. $=05^{\mathrm{h}} 07^{\mathrm{m}} 32.1^{\mathrm{s}}$ and Dec. $=-68^{\circ} 47^{\prime} 40.7^{\prime \prime}(\mathrm{J} 2000)$. The semi-major and semi-minor axes of $5.32^{\prime}\left( \pm 0.21^{\prime}\right)$ and $4.84^{\prime}\left( \pm 0.21^{\prime}\right)$, respectively, correspond to a size of $154.6 \times 140.8( \pm 6.1) \mathrm{pc}$ at the distance of the LMC $(50 \mathrm{kpc})$ with the major axis rotated $\sim 49.3^{\circ}$ East of North.

\subsection{Identification of point sources inside the shell}

In order to identify the point sources, we performed a maximumlikelihood source detection analysis on the XMM-Newton/EPIC images on fifteen images created from the three EPIC cameras in five energy bands as given in (Watson et al. 2009; Sturm et al. 2013). Source detection was performed simultaneously on all the images using the SAS task edetect_chain. Two point-like sources are found near the centre of the shell morphology. The source to the east is identified as a spectroscopically-confirmed quasar MQS J050736.44-684751.6 with $z=0.53$ (Souchay et al. 2012; Kozłowski et al. 2013) and is hence a background object which is projected in the line of sight of MCSNR J0507-6847.

The other point source marked with black cross in Fig. 1 and 
Table 1. XMM-Newton observations details of XMMU J050722.1-684758.

\begin{tabular}{ccccc}
\hline Date & ObsID & $\begin{array}{c}\text { Exposure } \\
\text { PN / MOS2 / MOS1 } \\
(\mathrm{ks})\end{array}$ & $\begin{array}{c}\text { Off-axis angle } \\
\text { PN / MOS2 / MOS1 }\end{array}$ & $\begin{array}{c}\text { Telecope vignetting } \\
\text { PN / MOS2 / MOS1 }\end{array}$ \\
\hline $2000 / 07 / 07$ & 0113000301 & $11.8 / 16.2 / 23.6$ & $10.4^{\prime} / 9.7^{\prime} / 8.7^{\prime}$ & $0.51 / 0.48 / 0.52$ \\
$2017 / 10 / 19$ & 0803460101 & $53.0 / 55.0 / 55.0$ & $10.0^{\prime} / 11.1^{\prime} / 11.1^{\prime}$ & $0.52 / 0.54 / 0.55$ \\
\hline
\end{tabular}

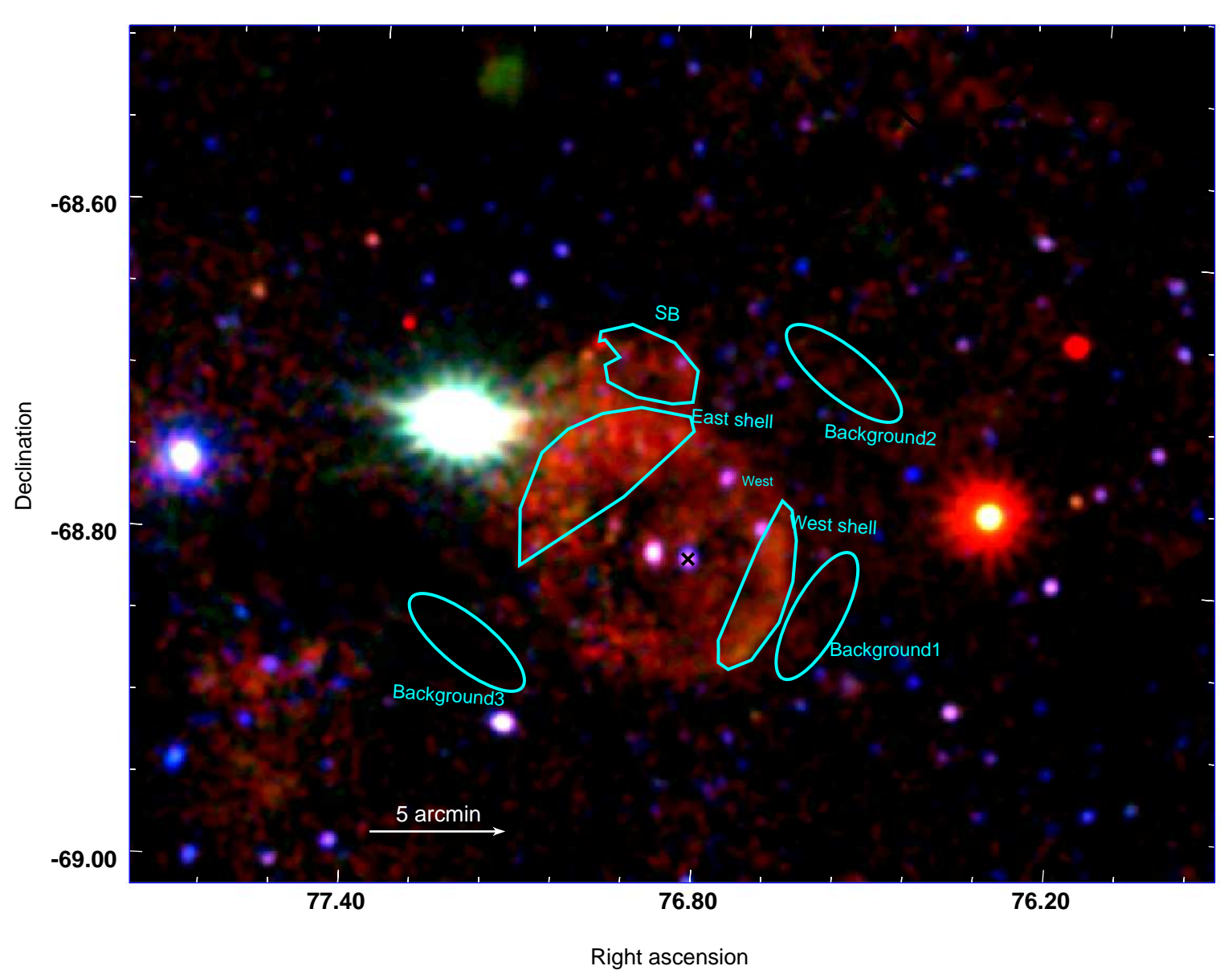

Figure 1. XMM-Newton EPIC RGB ( $\mathrm{R}=0.3-0.7 \mathrm{keV}, \mathrm{G}=0.7-1.1 \mathrm{keV}, \mathrm{B}=1.1-4.2 \mathrm{keV})$ image of XMMU J050722.1-684758. The regions used for spectral extraction are shown in cyan. The black cross shows the optical position of the BeXRB.

magenta cross in Fig. 2 lies $0.9^{\prime}$ away from the geometric centre of the SNR. Its best-determined position is R.A. $=05^{\mathrm{h}} 07^{\mathrm{m}} 22.37^{\mathrm{s}}$ and Dec. $=-68^{\circ} 47^{\prime} 58.2^{\prime \prime}(\mathrm{J} 2000)$ with a $1 \sigma$ statistical uncertainty of $0.72^{\prime \prime}$. The positional error is dominated by systematic astrometric uncertainties and a systematic error of $0.37^{\prime \prime}$ was added in quadrature (Rosen et al. 2016). The source was already identified as a BeXRB in the LMC from optical spectroscopic observations (BeCand 3 in van Jaarsveld et al. 2018). The confirmation of the nature of the source as a neutron star (see later section) and its positional proximity to the geometric centre of the SNR indicates that it is the compact object born out of the explosion of MCSNR J0507-6847. We calculated the probability of chance coincidence for a HMXB pulsar to lie within $0.9^{\prime}$ of the centre of an SNR within the LMC. For this we used the total of 21 known HMXBs with detected pulsations and $\sim 57$ confirmed SNRs (with sizes that can be resolved with
$X M M$-Newton) to be identified within the XMM-Newton observations of the LMC which cover an area of $\sim 20 \mathrm{deg}^{2}$ in total. Assuming that the HMXBs and SNRs are uniformly distributed within the survey area, the probability of finding a HMXB by chance within $0.9^{\prime}$ of the centre of an SNR is 0.04. It is to be noted that HMXBs and core-collapse SNRs (which constitute about $60 \%$ of all LMC SNRs, Maggi et al. 2016) are not uniformly distributed but follow star-forming regions, so this probability is likely underestimated. The probability of chance coincidence would also be slightly higher if all known HMXBs in the LMC are taken into account. The low probability supports the association of the HMXB with the SNR, but a chance coincidence can formally not be ruled out. 


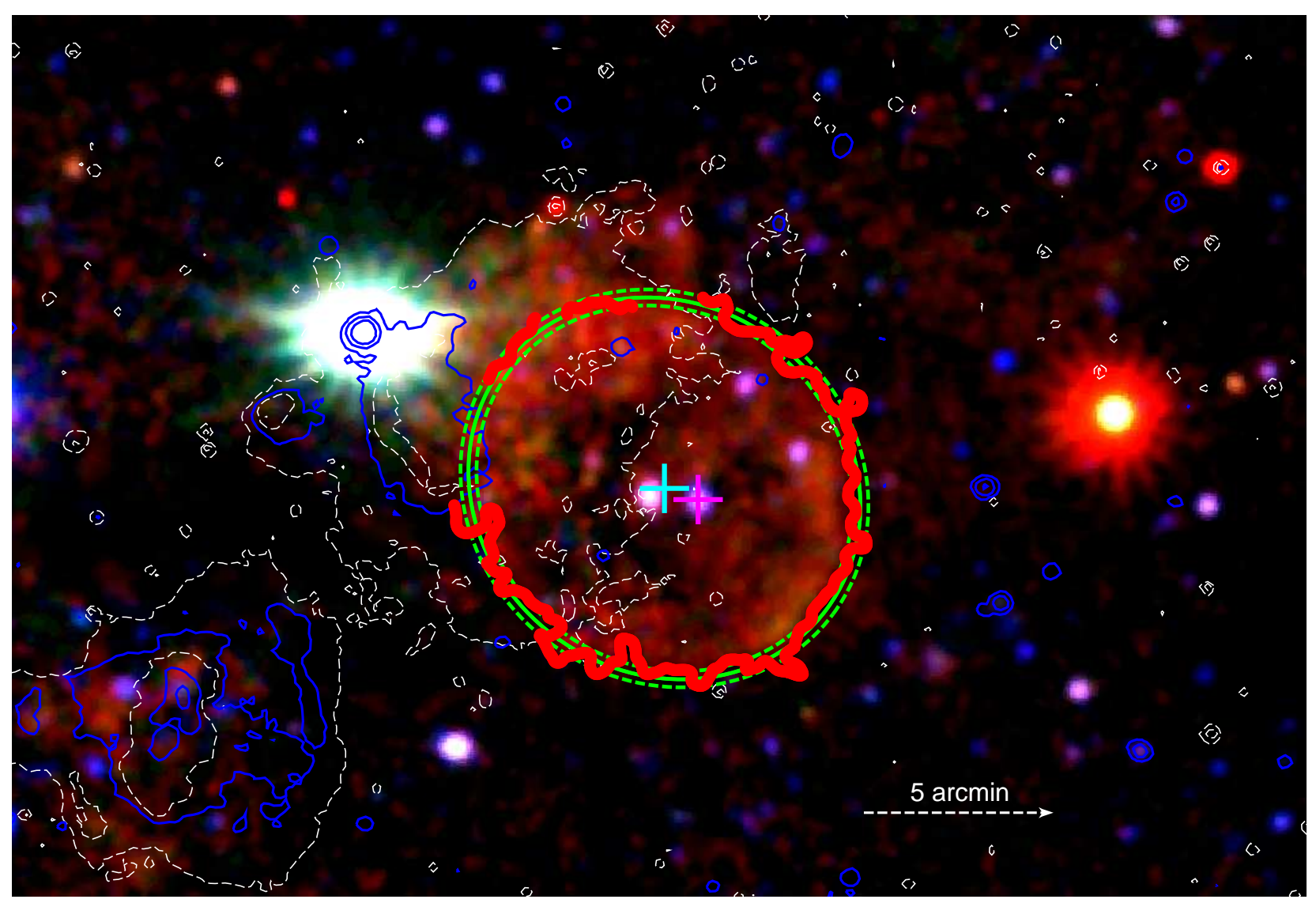

Figure 2. Zoomed in XMM-Newton EPIC RGB ( $\mathrm{R}=0.3-0.7 \mathrm{keV}, \mathrm{G}=0.7-1.1 \mathrm{keV}, \mathrm{B}=1.1-4.2 \mathrm{keV})$ image of XMMU J050722.1-684758. Overlaid in blue are radio contours from the latest Australian Square Kilometre Array Pathfinder (ASKAP) survey of the LMC at $888 \mathrm{MHz}$ (bandwidth is $288 \mathrm{MHz}$ ). The radio continuum contours correspond to 1,2 , and $3 \mathrm{mJy}_{\text {beam }}^{-1}$ while the image beam size is $13.7^{\prime \prime} \times 11.8^{\prime \prime}$ and local rms is $\sim 0.2 \mathrm{mJy}$ beam ${ }^{-1}$. The white line overlays the $\mathrm{H} \alpha$ image contours from the Magellanic Clouds Emission Line Survey (MCELS ) (Smith et al. 2004). The cyan cross shows the best-fit centre of the SNR and the magenta cross the position of the optical counterpart of the BeXRB. The red solid line indicate the X-ray contour level corresponding to $3 \sigma$ above the average background surface brightness. The green solid line shows the best-fit ellipse to the contour, with the dashed lines denoting the $1 \sigma$ errors on the best-fit.

\subsection{OGLE monitoring of the optical counterpart}

\subsubsection{Long-term OGLE I-band light curve}

Figure 3 shows the OGLE I-band light curve of the optical counterpart of XMMU J050722.1-684758 obtained during observing phases II-IV over a period of 13 years. The light-curve is variable in nature showing dip-like features, typical of Be stars with a binary companion. The average I band magnitude of the source is $15.8 \mathrm{mag}$. In order to verify the orbital period of the system, we detrended the light curve by subtracting a smoothed light curve (derived by applying a Savitzky-Golay filter with a window length of 101 data points, Savitzky \& Golay 1964) and computed Lomb-Scargle peridograms (Lomb 1976; Scargle 1982). A highly significant peak is found in the periodogram at 5.266 days with two other peaks at 1.235 and 40.16 days (Fig. 4). The 1.235 and 5.266 day periods (frequencies of 0.810 and $0.190 \mathrm{day}^{-1}$, respectively) are aliases of each other with the 1 day sampling period of the light curve. Since short periods near one day are most likely caused by non-radial pulsations of the Be star (e.g. Schmidtke et al. 2013), we suggest the 40.16 day period as the most likely orbital period of the system, which is also more typical for BeXRBs (Haberl \& Sturm 2016). The detrended light curve folded with that period is presented in Fig. 5.

\subsubsection{OGLE V-band and colour variations}

During OGLE phases III and IV also V-band measurements of the optical counterpart of XMMU J050722.1-684758 are available, in particular around the sharp drop in brightness around MJD $56200 \mathrm{~d}$ (Figs. 3 and 6). We created I-V colour indices by using the measured V-band magnitudes and neighbouring I-band values interpolated to the time of the V-band measurement. The colour - magnitude diagram is shown in Fig. 7.

\subsection{X-ray timing analysis}

XMMU J050722.1-684758 displayed a net count rate (PN) of 0.03 c/s during Obs. 1 and $0.05 \mathrm{c} / \mathrm{s}$ during Obs. 2 in the energy range of $0.2-12 \mathrm{keV}$ confirming its variable nature. The X-ray light curves during the individual observations however did not exhibit variability on shorter time scales. Since the source was brighter during Obs. 2 and was observed for a longer duration, data from this observation was considered to study the temporal properties of the source in detail. To look for a possible periodic signal in the X-ray light curve of the HMXB, we extracted source events using a circular region with radius $26^{\prime \prime}$ centred on the best-fit position, and a background region of a larger size away from the source as shown in Fig. 1. The light curve was corrected for all effects like vignetting and Point Spread 


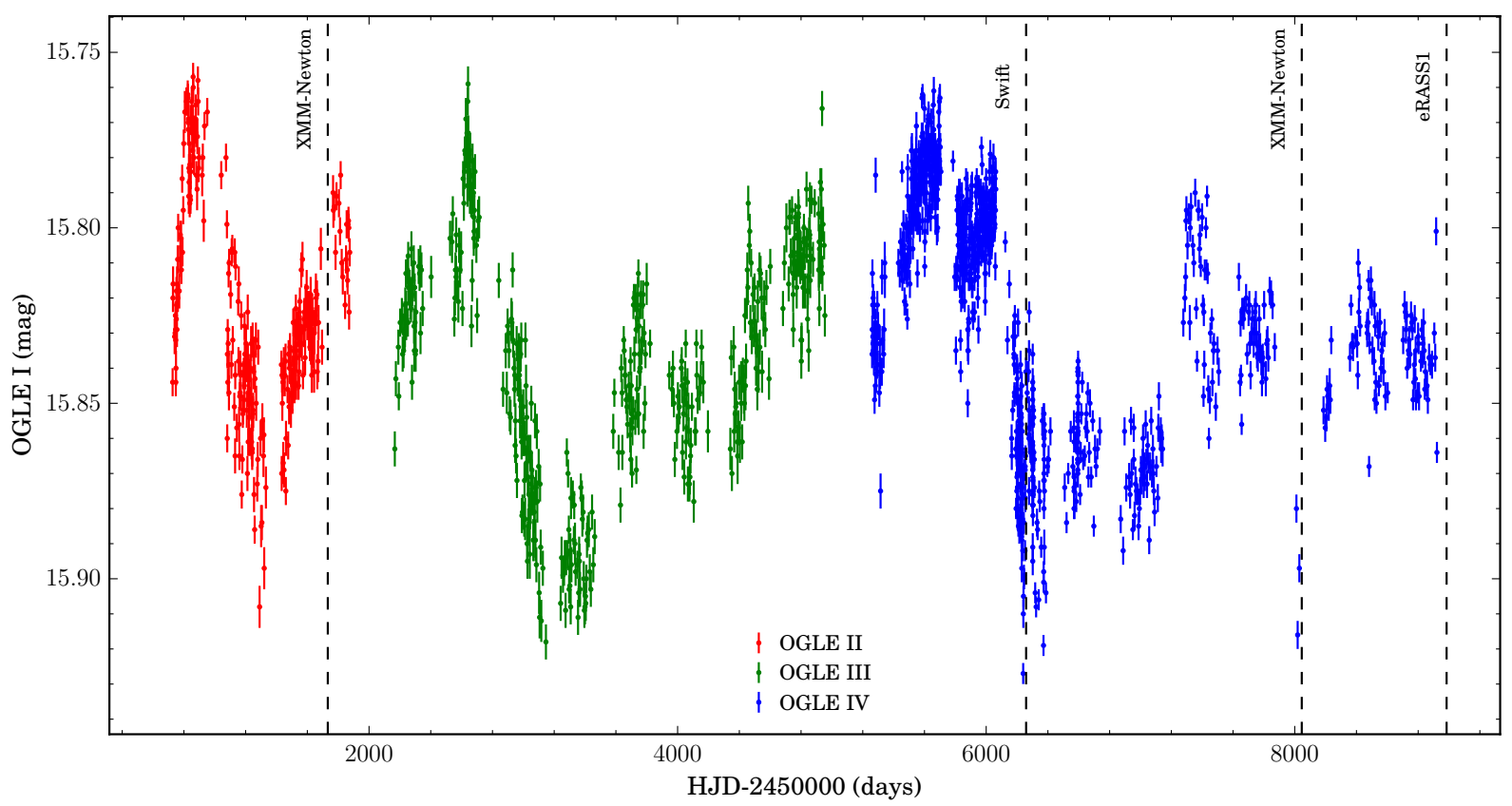

Figure 3. OGLE I-band light curve of 2MASS 05072214-6847592 from October 1997 to March 2020. Epochs of X-ray observations analysed in this work are marked with vertical dashed lines.

Table 2. Swift observations details of XMMU J050722.1-684758.

\begin{tabular}{|c|c|c|c|c|}
\hline Date & ObsID & Mode & $\begin{array}{l}\text { Exposure } \\
\text { (s) }\end{array}$ & $\begin{array}{c}\text { Observed } \\
\text { Luminosity }\end{array}$ \\
\hline 55962.8 & 00045577002 & $\mathrm{PC}$ & 2128 & $<8.6$ \\
\hline 55994 & 00045444002 & $\mathrm{PC}$ & 248 & $<77.3$ \\
\hline 56252.5 & 00045444003 & $\mathrm{PC}$ & 779 & $<37.8$ \\
\hline 56254.9 & 00045444004 & $\mathrm{PC}$ & 904 & $<28.8$ \\
\hline 56259.3 & 00045444005 & $\mathrm{PC}$ & 1116 & 56.7 \\
\hline 56377.8 & 00045576001 & $\mathrm{PC}$ & 2872 & $<6.9$ \\
\hline 56968.9 & 00033486001 & $\mathrm{PC}$ & 961 & $<14.4$ \\
\hline 56972.2 & 00033486002 & $\mathrm{PC}$ & 434 & $<31.8$ \\
\hline 57109.2 & 00033486003 & $\mathrm{PC}$ & 439 & $<31.5$ \\
\hline 57653 & 00034730001 & $\mathrm{PC}$ & 1101 & $<18.4$ \\
\hline 58220.5 & 00094089001 & $\mathrm{PC}$ & 247 & $<55.9$ \\
\hline 58234.3 & 00094089003 & $\mathrm{PC}$ & 243 & $<57.1$ \\
\hline 58255.6 & 00094089004 & $\mathrm{PC}$ & 245 & $<56.5$ \\
\hline 58262.3 & 00094089005 & $\mathrm{PC}$ & 247 & $<55.9$ \\
\hline 58290.9 & 00094089007 & $\mathrm{PC}$ & 245 & $<56.5$ \\
\hline 58332.6 & 00094089009 & $\mathrm{PC}$ & 250 & $<55.4$ \\
\hline 58346.8 & 00094089010 & $\mathrm{PC}$ & 245 & $<56.5$ \\
\hline 58360.7 & 00094089011 & $\mathrm{PC}$ & 232 & $<59.5$ \\
\hline 58374.7 & 00094089012 & $\mathrm{PC}$ & 250 & $<55.4$ \\
\hline 58388.5 & 00094089013 & $\mathrm{PC}$ & 240 & $<57.7$ \\
\hline 58416.8 & 00094089014 & $\mathrm{PC}$ & 242 & $<57.1$ \\
\hline 59006.9 & 00034730003 & $\mathrm{PC}$ & 1925 & $<11.6$ \\
\hline
\end{tabular}

Luminosity in the $0.5-10 \mathrm{keV}$ band in units of $10^{34} \mathrm{erg} \mathrm{s}^{-1}$. To convert Swift/XRT count rates to luminosities we used the spectral parameters obtained from the fit to the XMM-Newton data. The conversion factor is $2.1 \times 10^{37} \mathrm{erg} \mathrm{s}^{-1}(\mathrm{c} / \mathrm{s})^{-1}$. Only Swift exposures of $\geq 200 \mathrm{~s}$ covering the source have been used for this purpose.

Function losses by the task epiclccorr. At first, we searched for a periodic signal in the barycentre-corrected XMM-Newton EPIC light curve in the energy range above $1 \mathrm{keV}$ using a Lomb-Scargle periodogram analysis in the period range of 0.5-3000 s (Lomb 1976;

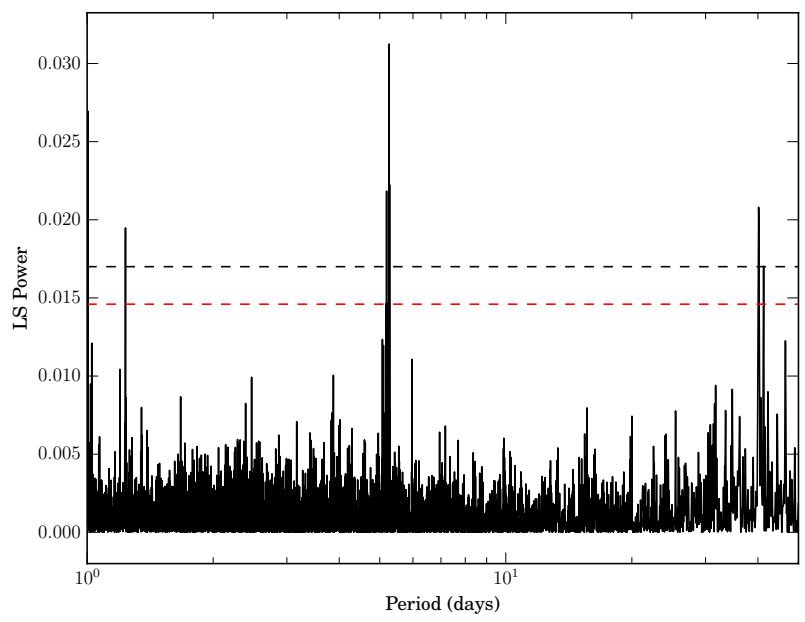

Figure 4. Lomb-Scargle periodogram of the detrended OGLE I-band light curve of 2MASS 05072214-6847592. Significant peaks are found at 1.235, 5.266 and 40.16 days. The red and black dashed lines mark the $95 \%$ and $99 \%$ confidence levels, respectively.

Scargle 1982). A strong periodic signal is detected at $570.4 \mathrm{~s}$ indicating the spin period of the neutron star in the BeXRB (Fig. 8). In order to determine the pulse period more precisely, we employed the Bayesian periodic signal detection method described by Gregory \& Loredo (1996). The spin period and its associated $1 \sigma$ error are determined to $570.35 \pm 0.35 \mathrm{~s}$. The spin period and its associated $X M M$-Newton EPIC light curve in the range of $1-12 \mathrm{keV}$, folded with the best-obtained period is shown in Fig. 9. The pulse fraction in the same energy range is $40 \%$ and no change in the pulse shape or pulse fraction can be detected within this energy range. Pulsations cannot be detected below $1 \mathrm{keV}$, possibly due to the contamination from the SNR.

The source was scanned 49 times during the first all-sky survey (eRASS1) of the eROSITA instrument (Predehl et al. 2020) on board 


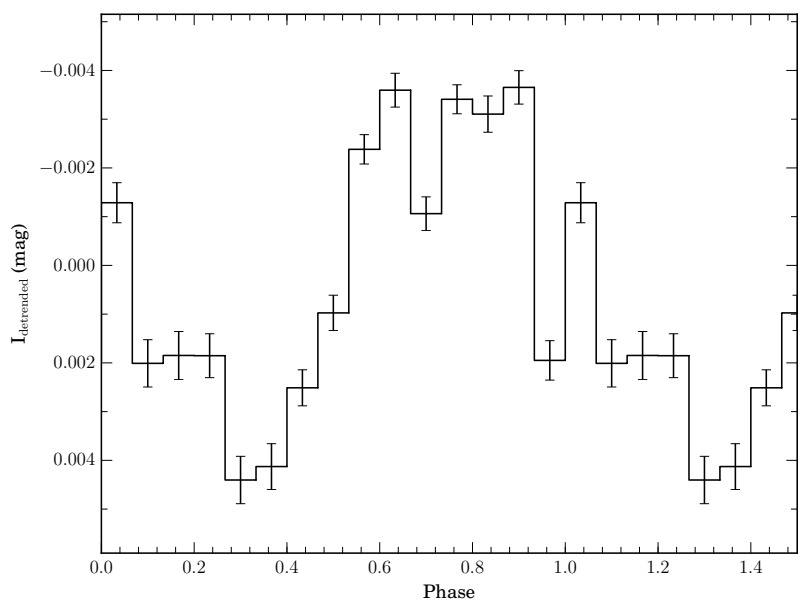

Figure 5. Detrended OGLE I-band light curve folded with a period of 40.1609 days.

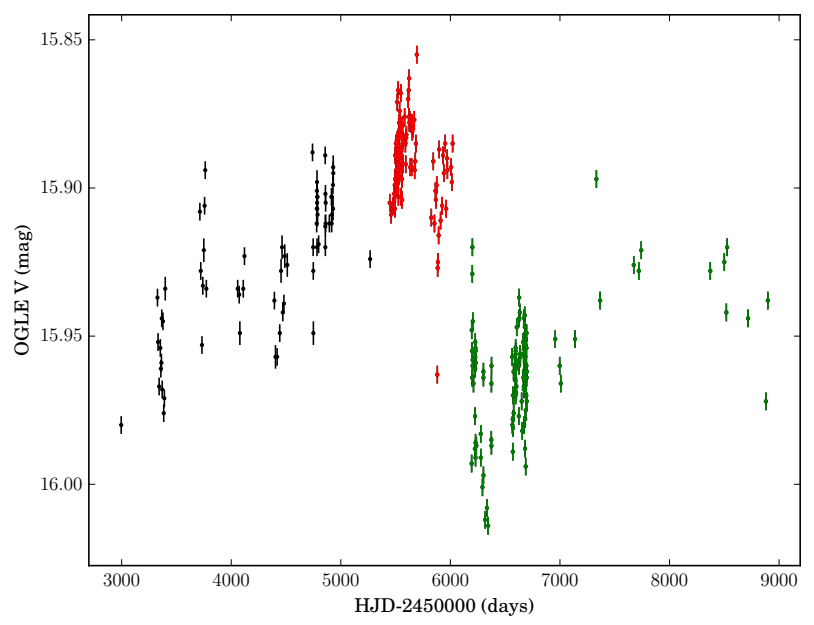

Figure 6. OGLE V-band light curve of 2MASS 05072214-6847592. The colours mark different phases of brightness and colour evolution as shown in Fig. 7.

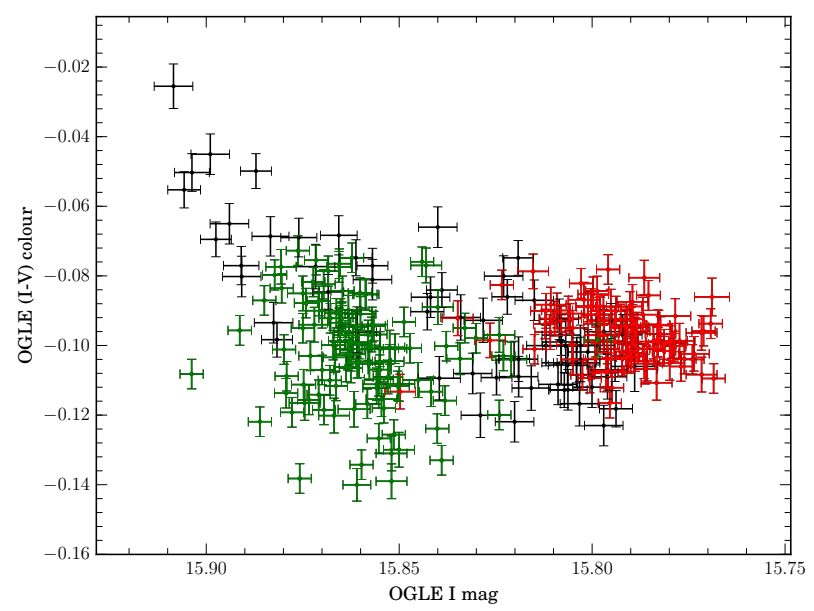

Figure 7. OGLE colour (I-V) - magnitude (I) diagram of 2MASS 05072214-6847592. During brightness rise (black) the emission becomes redder reaching asymptotically a minimum value in I-V (red), which is also maintained after the brightness drop (green).

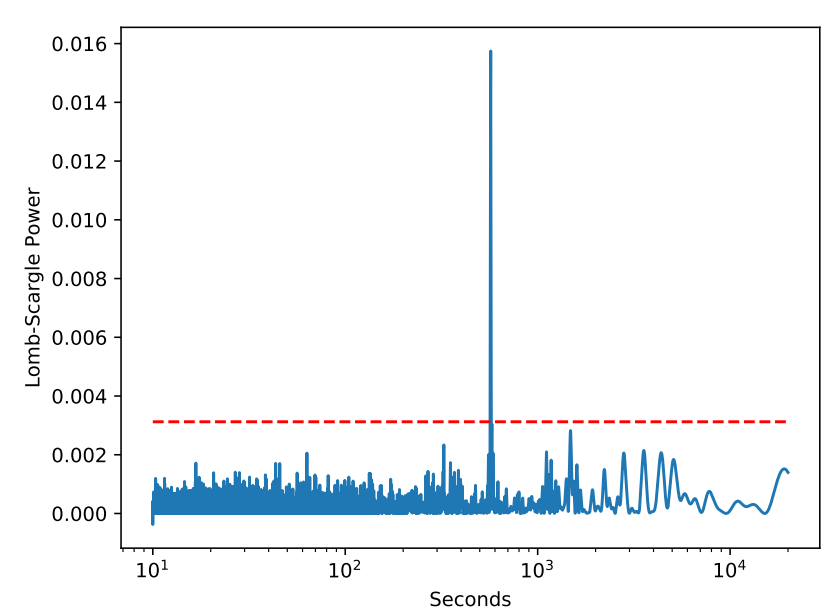

Figure 8. Lomb-Scargle periodogram of the XMM-Newton EPIC light curve in the energy band of 1-12 keV (ObsID 0803460101). The peak indicates the spin period of the neutron star. The red dashed line mark the $99.73 \%$ confidence levels.

the Russian/German Spektrum-Roentgen-Gamma (SRG) mission. The scans spanned between MJD 58980.53 to MJD 58987.09, accumulating a total exposure of $1543 \mathrm{~s}$. The source was variable during the scans and the count rates remained around $0.08 \pm 0.03 \mathrm{c} / \mathrm{s}(0.2-$ $8.0 \mathrm{keV}$ ) around MJD 58981.54 in the beginning and dropped to $<0.01 \mathrm{c} / \mathrm{s}$ towards the end of the eRASS1 scans. Source detection was performed simultaneously on all the images in the standard eROSITA energy bands of $0.2-0.6 \mathrm{keV}, 0.6-2.3 \mathrm{keV}$ and $2.3-$ $5.0 \mathrm{keV}$. The vignetting and Point Spread Function corrected count rate in the energy range of $0.2-5.0 \mathrm{keV}$ was $0.05 \pm 0.01 \mathrm{c} / \mathrm{s}$ indicating a similar average flux as observed during XMM-Newton Obs. 2.

\subsection{X-ray spectral analysis}

For the spectral analysis, the SAS tasks rmfgen and arfgen were used to create the redistribution matrices and ancillary files. The significant extent of the SNR and varying off-axis position in the two observations was taken into account by extracting spectra from vignetting-weighted event lists, created through the SAS task evigweight (as described in Maggi et al. 2016). To account for the spatially-dependent non-X-ray background (NXB) in extended emission spectra, spectra were extracted from Filter Wheel Closed (FWC) data at the same detector position. Spectra were binned to achieve a minimum of 20 and 25 counts per spectral bin for the point source and SNR, respectively, to allow using the $\chi^{2}$-statistic. The spectral analysis was performed using the XSPEC fitting package, version 12.9 (Arnaud 1996). Errors were estimated at the 90 percent confidence level, unless otherwise stated.

\subsubsection{MCSNR J0507-6847}

Spectra were extracted from the entire shell region, from the eastern and western hemispheres, and from the superbubble region to the North (see Fig. 1). Background spectra were accumulated in nearby regions chosen individually per instrument and observation. In Obs. 1 we also made sure to exclude a large region around the bright SNR N103B and its streak of out-of-time events. Point sources detected in the background and shell region, including the background AGN and HMXB, were excised using circular regions 
Table 3. X-ray spectral parameters of MCSNR J0507-6847

\begin{tabular}{|c|c|c|c|c|c|c|c|c|c|}
\hline Region & $\begin{array}{c}N_{H}^{\mathrm{LMC}} \\
\left(10^{20} \mathrm{~cm}^{-2}\right)\end{array}$ & $\begin{array}{c}k T \\
(\mathrm{keV})\end{array}$ & $\begin{array}{c}\tau=n_{e} t \\
\left(10^{11} \mathrm{~cm}^{-3} \mathrm{~s}^{-1}\right)\end{array}$ & $\mathrm{O}$ & $\mathrm{Ne}$ & $\mathrm{Mg}$ & $\mathrm{Fe}$ & $\begin{array}{c}\mathrm{EM}^{\mathrm{a}} \\
\left(10^{57} \mathrm{~cm}^{-3}\right)\end{array}$ & $\chi^{2} / \operatorname{dof}\left(\chi_{r}^{2}\right)$ \\
\hline \multicolumn{10}{|c|}{ vapec parameters } \\
\hline Shell & $7.1_{-3.5}^{+4.2}$ & $0.25 \pm 0.01$ & - & $0.27_{-0.07}^{+0.10}$ & $0.38_{-0.10}^{+0.16}$ & $0.63_{-0.24}^{+0.37}$ & $0.12_{-0.03}^{+0.05}$ & $1.21_{-0.38}^{+0.48}$ & $2156.4 / 2051(1.05)$ \\
\hline East & $0.8(<5.5)$ & $0.22 \pm 0.01$ & - & & & - & & $0.98_{-0.11}^{+0.29}$ & $154.7 / 140(1.11)$ \\
\hline West & $3.3(<12.7)$ & $0.23 \pm 0.01$ & - & & & - & & $0.89_{-0.18}^{+0.40}$ & $225.3 / 213(1.06)$ \\
\hline SB & $0.0(<7.6)$ & $0.23 \pm 0.03$ & - & & & - & & $0.53 \pm 0.12$ & $92.5 / 88(1.05)$ \\
\hline \multicolumn{10}{|c|}{ vpshock parameters ${ }^{b}$} \\
\hline Shell & $0.0(<1.3)$ & $0.36_{-0.03}^{+0.07}$ & $6.3_{-3.4}^{+8.1}$ & $0.30_{-0.05}^{+0.04}$ & $0.40_{-0.03}^{+0.07}$ & $0.44 \pm 0.16$ & $0.11 \pm 0.02$ & $0.49_{-0.12}^{+0.13}$ & $2148.6 / 2050(1.05)$ \\
\hline East & $0.0(<1.6)$ & $0.77_{-0.21}^{+0.43}$ & $0.3_{-0.1}^{+0.2}$ & & & - & & $0.14_{-0.02}^{+0.03}$ & $149.9 / 139(1.08)$ \\
\hline $\mathrm{SB}$ & $0(<39.9)$ & $0.88_{-0.46}^{+1.57}$ & $0.2_{-0.1}^{+0.6}$ & & & - & & $0.07_{-0.02}^{+0.51}$ & $90.2 / 87(1.04)$ \\
\hline
\end{tabular}

a The emission measure $E M=n_{e} n_{\mathrm{H}} V$, the product of electronic and proton densities with the total emitting volume, acts as a (temperature-dependent) normalisation of thermal plasma models.

${ }^{\mathrm{b}}$ The ionisation time scale could not be constrained for the West region, and thus we do not report vpshock parameters for it.

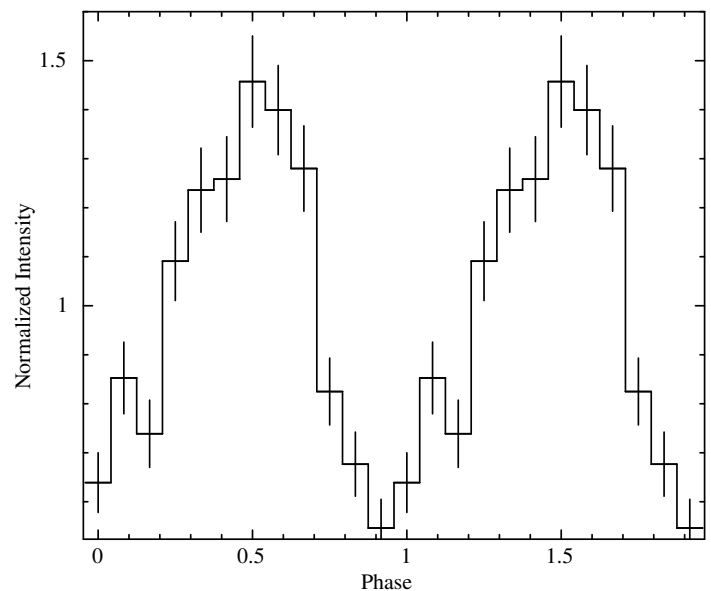

Figure 9. Corrected EPIC-PN light curve folded with $570.35 \mathrm{~s}$, showing the pulse profile of the HMXB in the energy band of $1-12 \mathrm{keV}$.

with a 25 "radius. Thus, we estimated that $<5 \%$ of the source counts from these sources will contaminate our extended source spectra due to PSF leaks. Source and background spectra were fitted simultaneously, with NXB parameters tied to those obtained from the FWC spectra, and astrophysical X-ray background (AXB) parameters constrained from background spectra. The AXB comprises Galactic thermal emission (Local Hot Bubble, Galactic halo), cosmic X-ray background, and LMC diffuse emission. More details on this method can be found in Maggi et al. (2016, 2019).

The X-ray absorption was modeled using the tbabs model (Wilms et al. 2000) with atomic cross sections adopted from Verner et al. (1996). We used two absorption components: The first one to describe the Galactic foreground absorption, where we used a fixed column density of $6 \times 10^{20} \mathrm{~cm}^{-2}$ (Dickey \& Lockman 1990) with abundances taken from Wilms et al. (2000). The second component accounts for the LMC material in front of the object. For the latter absorption component, the abundances were set to LMC abundances following Maggi et al. (2016) and the column density $N_{H}^{\mathrm{LMC}}$ was free in the analysis. The cosmic X-ray background, seen through all the LMC, was absorbed by the total line-of-sight column density

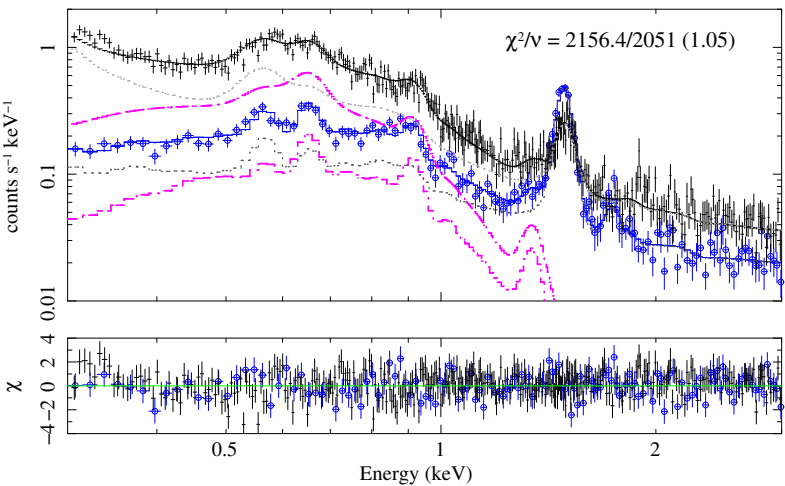

Figure 10. X-ray spectra of the SNR MCSNR J0507-6847. The upper panel shows the simultaneous spectral fit using all the available data (Table 1). For the sake of clarity only PN and MOS2 data from obsid 0803460101 are displayed in black dots and blue circles, respectively. The total background models for PN and MOS are shown by the dotted gray lines. The best-fit vapec model for the SNR emission is shown by the dashed magenta lines, as convolved with the PN and MOS spectral responses (top and bottom curves, respectively). The lower panel displays the residuals after the fit.

of $2.0 \times 10^{21} \mathrm{~cm}^{-2}$, obtained from the ATCA+Parkes map of Kim et al. (2003) averaged over 0.1 squared degree.

We fit the X-ray spectra with collisional ionisation equilibrium and non-equilibrium ionisation thermal emission models (XSPEC models vapec and vpshock, respectively), typical for shock-heated plasma in SNRs. We also attempted to fit the abundances of the main elements in the energy range of the SNR emission, namely $\mathrm{O}, \mathrm{Ne}, \mathrm{Mg}$, and $\mathrm{Fe}$. However, meaningful constraints were only obtained for the entire shell spectrum and not for the smaller regions due to limited statistics. For these spectra and all other elements, abundances were fixed to that of the LMC hot gas. Best-fit spectral parameters are listed in Table 3. Improvements to the fit quality with the non-equilibrium ionisation model were only marginal. Regardless of ionisation equilibrium status, the overall absorption is low, indicating an object on the nearer side of the LMC gaseous disk. As expected from the $\mathrm{X}$-ray images and dearth of emission at $E \gtrsim 1 \mathrm{keV}$, the plasma temperature is relatively low, at $k T<0.4 \mathrm{keV}$.

No strong spatial variations can be identified, all the parameters 


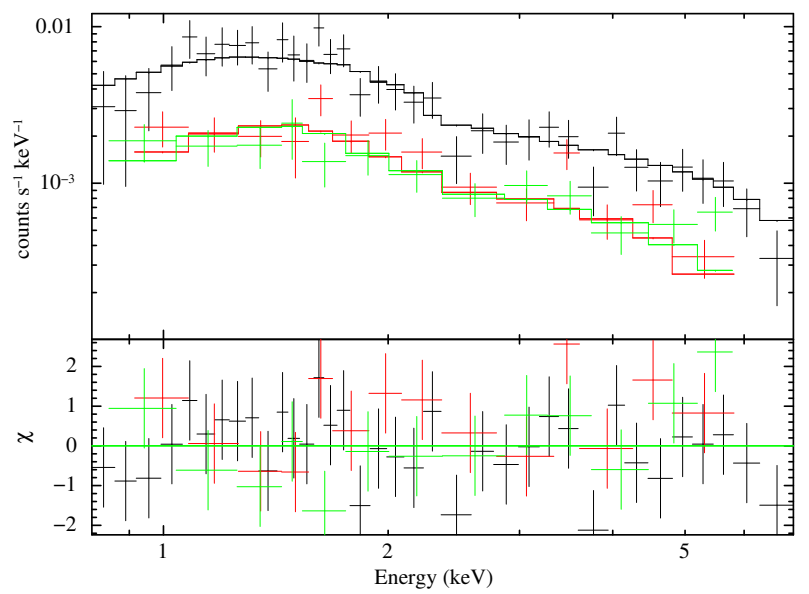

Figure 11. EPIC spectra of the HMXB XMMU J050722.1-684758. Black, red and green denotes PN, MOS1 and MOS2 data points and model (histogram), respectively. The spectra have been rebinned for visual clarity.

being consistent between eastern and western part of the shell, within the larger uncertainties stemming from the reduced statistics of the smaller regions. The emission from the superbubble region cannot be distinguished as well for the same reason as given above. With both the vapec and vpshock models, the abundances of $\mathrm{O}$, $\mathrm{Ne}, \mathrm{Mg}$, and $\mathrm{Fe}$ and their ratios are the same: They are those of the LMC hot gas phase (Maggi et al. 2016; Schenck et al. 2016) and reveal no enhancement by $\mathrm{SN}$ ejecta.

The total observed luminosity in the $0.3-8 \mathrm{keV}$ band is $2.2( \pm 0.06) \times 10^{35} \mathrm{erg} \mathrm{s}^{-1}$, lower than reported in Chu et al. (2000), likely because we measured a slightly lower plasma temperature and higher $N_{H}$ (with better spectral resolution than ROSAT) and excluded point sources previously unresolved. This sets MCSNR J0507-6847 among the brightest third of LMC SNRs (Maggi et al. 2016). However, given the large extent, the source is in the $20 \%$ faintest SNRs when comparing surface brightness, which is the limiting factor for the study of extended sources.

\subsubsection{HMXB}

As in the case of the X-ray timing analysis, only data from Obs. 2 were of sufficient statistical quality to perform a detailed spectral analysis. The X-ray spectrum of the HMXB XMMU J050722.1-684758 contains some contribution from the overlapping extended SNR as can be seen from Fig. 1. In order to model this contribution, we included a component for the SNR emission in the spectral fit with the normalisation component left free - since the surface brightness of the SNR is not uniform, we do not constrain the contamination level to the fractional geometric area covered by the source extraction region. The X-ray spectrum of the HMXB can be satisfactorily modelled with an absorbed powerlaw. The absorption scheme was the same as for the SNR, but the column density for the HMXB, $N_{H}^{\text {local }}$, represents both LMC gas in front of the source and potential local contributions.

The spectral parameters for the best-fit model using an absorbed power law are listed in Table 4 and the spectra and best-fit model are shown in Fig. 11. The source was detected with an average absorption-corrected luminosity of $9 \times 10^{34} \mathrm{erg} \mathrm{s}^{-1}$. Assuming that the source exhibited a similar spectrum during Obs.1 and from the PN count rate, we estimated an absorption corrected luminosity of $7 \times 10^{34} \mathrm{erg} \mathrm{s}^{-1}$ for XMMU J050722.1-684758 during Obs. 1 .

The local absorption component $N_{H}^{\text {local }}$ measured in the case
Table 4. X-ray spectral parameters of the HMXB XMMU J050722.1-684758 for a power-law model.

\begin{tabular}{lc}
\hline Parameter & Value \\
\hline$N_{H}^{\text {local }}\left(10^{22} \mathrm{~cm}^{-2}\right)$ & $0.6_{-0.3}^{+0.4}$ \\
$\Gamma$ & $1.2 \pm 0.2$ \\
Flux $^{a}(0.2-12.0 \mathrm{keV})$ & $2.6 \pm 0.3$ \\
Flux (unabsorbed) $^{a}(0.2-12.0 \mathrm{keV})$ & $3.0 \pm 1.0$ \\
Absorption corrected X-ray luminosity $^{a}\left(\mathrm{erg} \mathrm{s}^{-1}\right)$ & $8.5 \pm 1.0 \times 10^{34}$ \\
\hline$\chi^{2}$ & 58.02 \\
Degrees of Freedom $^{2}$ & 59 \\
\hline
\end{tabular}

${ }^{a}$ Flux in units of $10^{-13} \mathrm{erg} \mathrm{cm}^{-2} \mathrm{~s}^{-1}$ and assuming a distance of $50 \mathrm{kpc}$ in the energy band of $0.2-12 \mathrm{keV}$.

${ }^{b}$ Absorption in units of $10^{22} \mathrm{~cm}^{-2}$ Line-of-sight Galactic absorption was fixed to $6 \times 10^{20} \mathrm{~cm}^{-2}$

${ }^{c}$ Errors are quoted at $90 \%$ confidence.

of XMMU J050722.1-684758 is significantly higher than that measured for the SNR (see Table 4). In order to estimate the total amount of absorption column density towards the direction of the source, we extracted the spectrum from the quasar MQS J050736.44-684751.6 which overlaps with the SNR along the line of sight (Fig. 1). The spectrum was well-fitted by an absorbed power-law taking into account the determined redshift of the source. The best-fit parameters correspond to $\Gamma=2.3_{-0.3}^{+0.2}$ and $\mathrm{N}_{\mathrm{H}}=3 \times 10^{21} \mathrm{~cm}^{-2}$. This is only slightly higher than the average integrated LMC column $\left(2.0 \times 10^{21} \mathrm{~cm}^{-2}\right.$, Sect. 3.5 .1$)$, perhaps due to further contribution by the host galaxy or intrinsic to the AGN. Nevertheless, this is several times lower than estimated from the HMXB XMMU J050722.1-684758 which is very close to the quasar in projection. This establishes that the absorption of XMMU J050722.1-684758 is not originating from the LMC largescale structure. Instead, it indicates that $N_{H}^{\text {local }}$ is dominated by a local absorbing column density as is often detected in the case of HMXBs in the MCs (Vasilopoulos et al. 2013; Coe et al. 2015; Maitra et al. 2018).

\section{DISCUSSION}

We report here the positional coincidence of the BeXRB XMMU J050722.1-684758 near the geometrical centre of MCSNR J0507-6847 and the possible association of the two sources. We performed detailed timing and spectral analysis of the BeXRB using XMM-Newton, Swift, eROSITA, and OGLE data and confirmed its nature as a highly magnetized neutron star. We also performed detailed X-ray spectral analysis of MCSNR J0507-6847 using XMM-Newton observations and compared with optical and radio observations to understand its nature.

\subsection{Nature of XMMU J050722.1-684758: a BeXRB pulsar in the LMC}

Using the latest $X M M$-Newton observation where the source was in the field of view (Obs.2) we discovered highly significant pulsations at 570 s which confirm the nature of XMMU J050722.1-684758 as a new BeXRB pulsar in the LMC. A total of $\sim 60$ (candidate) HMXBs are known in the LMC out of which $\sim 90 \%$ are Be/X-ray binaries and the rest are supergiant systems (Maitra et al. 2021; Haberl et al. 2020; Maitra et al. 2020, 2019; van Jaarsveld et al. 2018; Vasilopoulos et al. 2018; Antoniou \& Zezas 2016). Pulsations 
have been detected from only a small fraction of them (21) until now. This source significantly improves our knowledge of BeXRB pulsars in the LMC especially due to its possible association with SNR MCSNR J0507-6847 which allows an estimate of its age. The $\mathrm{X}$-ray intensity varies on long time scales as seen by comparing the Swift observations in Table 2. The eRASS1 scans also displayed variability on shorter timescales.

The optical light curve of XMMU J050722.1-684758 in the I-band spanning 13 years is highly variable in nature showing diplike features as is typical for Be stars with binary companion. An intriguing fact is that the XMM-Newton Obs. 2 and the Swift detection fall into 'dips" in the optical emission as seen from the I-band light curve. The I vs. V-I colour-magnitude evolution displays that the emission becomes redder during the rise of the optical brightness, and asymptotically reaches a minimum value in I-V color. This indicates that as the Be disc grows in size, the optical emission gets brighter while the red continuum increases, a behaviour similar to what is seen in other Magellanic Cloud BeXRBs (e.g. Haberl et al. 2017; Vasilopoulos et al. 2014; Coe et al. 2012)

\subsection{Nature and properties of MCSNR J0507-6847}

The shell-like morphology of MCSNR J0507-6847 combined with its X-ray spectrum is typical of an middle-aged to old SNR. What sets it apart is its extreme diameter of $150 \mathrm{pc}$, easily the largest when compared to the population of confirmed LMC SNRs (the largest SNR in Bozzetto et al. 2017, is $128 \mathrm{pc} \times 82 \mathrm{pc}$ ). Combined with the lack of obvious shell counterparts in optical and radio, the most natural explanation of the properties of MCSNR J0507-6847 is that it is indeed a supernova remnant, but expanding into a very tenuous environment.

One can estimate properties such as ambient density, explosion energy, and age of the SNR based on its morphological and spectral parameters, under the assumption that it is in the Sedov phase (e.g. van der Heyden et al. 2004; Maggi et al. 2012). The electronic density $n_{e}$ is calculated from the emission measure (Table 3 ) and corresponding emitting volume $V$. For the latter we assume a spherical volume with a radius $R_{\mathrm{av}}$ averaged between semi-major and semi-minor axes. It results in $n_{e}=1.9( \pm 0.4) \times 10^{-3} f^{-1} \mathrm{~cm}^{-3}$ for the vapec model, and $n_{e}=1.2( \pm 0.1) \times 10^{-3} f^{-1} \mathrm{~cm}^{-3}$ for the vpshock model, with $f$ the filling factor of the plasma within the volume $(f \leq 1)$. Thus, it is clear that MCSNR J0507-6847 is expanding in a rarefied medium. Still, given the size of the shell, the total swept-up mass $M_{\mathrm{sw}} \propto n_{e} V$ within it is large, ranging from $881( \pm 190) f^{2} M_{\odot}$ to $M_{\mathrm{SW}}=557( \pm 54) f^{2} M_{\odot}$ for the vapec and vpshock models, respectively. Even accounting for a small filling factor $f, M_{\mathrm{sw}}$ is much in excess of the ejecta and circumstellar material mass for any type of progenitor, and is therefore dominated by ambient ISM, justifying the assumption of a Sedov phase. The SN explosion energy is set by the size of the shell $R$, the plasma temperature $k T_{S}$ (which depends on the shock speed), and the ambient density as $E_{0} \propto k T_{S} R_{\mathrm{av}}^{3} n_{e}$. We found $E_{0}=1.0( \pm 0.3) \times 10^{51} f^{-1} \mathrm{erg}$ and $E_{0}=0.9( \pm 0.2) \times 10^{51} f^{-1}$ erg for the vapec and vpshock models, respectively. This is close to the canonical $10^{51}$ erg for a $\mathrm{SN}$ and thus consistent with the interpretation of the energetics of MCSNR J0507-6847 being dominated by a single SN. Finally, the dynamical age of MCSNR J0507-6847 $t_{\text {dyn }} \propto R_{\mathrm{av}}\left(k T_{s}\right)^{-1 / 2}$ is in the range 55 to $63 \mathrm{kyr}$ in the collisional ionisation equilibrium case, and between 43 and $54 \mathrm{kyr}$ for the non-equilibrium case, as the temperature is slightly higher. MCSNR J0507-6847 is one of the oldest LMC SNRs, as expected at such a large size. The derived ambient density, explosion energy and age of the SNR are consistent with the estimates of Chu et al. (2000).

Given the large size, low density, and complete shell morphology, Chu et al. (2000) suggested that the SNR was located in the near side of the LMC halo. Here we suggest another possibility. The NGC 1850 cluster, which is located at the same position is a double cluster comprising a large globular-like cluster separated from a smaller, younger compact cluster, known as NGC 1850B, by $\sim 30^{\prime \prime}$ (e.g. Robertson 1974; Fischer et al. 1993; Gilmozzi et al. 1994). The ages of both clusters have been determined in various studies. Gilmozzi et al. (1994) determined ages of $50 \pm 10 \mathrm{Myr}$ and $4.3 \pm 0.9 \mathrm{Myr}$ for the main cluster and NGC 1850B, respectively. Other studies are in general agreement, with estimates ranging from 40-100 Myr for the main cluster and 1-10 Myr for NGC 1850B (e.g. Fischer et al. 1993; Vallenari et al. 1994). Mass estimates for NGC 1850 are $\sim 10^{4-5} \mathrm{M}_{\odot}$ (Fischer et al. 1993; McLaughlin \& van der Marel 2005), placing it among the most massive in the LMC outside 30 Dor. Given the age of the main NGC 1850 cluster, the massive stellar population has already been lost to $\mathrm{SNe}$, and there are no stars remaining that are capable of photoionising the N103 shell. Rather, the young massive stars of NGC 1850B are responsible (Fischer et al. 1993; Ambrocio-Cruz et al. 1997). However, in its infancy, the main NGC 1850 cluster would have been a powerhouse, containing a significant massive stellar population, more than capable of driving a large superbubble into the ISM. To explain the low ambient density inferred for the SNR, we propose the following scenario: $(i)$ the main NGC 1850 cluster created and powered a superbubble in the region; (ii) the evolution of this superbubble stalled after $\sim 40 \mathrm{Myr}$ when the massive stellar population was lost and the internal pressure dropped below the ISM pressure, leaving the large, low density superbubble relic; (iii) NGC 1850B formed near the main cluster and its massive stars are now photoionising part of the original superbubble shell, which we know as N103; (iv) the SNR progenitor exploded in the interior of the superbubble relic created by the NGC 1850 main cluster. We also note that NGC 1850 and N103 are projected against the inside edge of the supergiaint shell SGS5 (Kim et al. 1999) and the initial superbubble could have blown out into this region. In any case, the proposed scenario can explain the very low ambient density into which the SNR has evolved.

\subsection{A new BeXRB-SNR association ?}

Using ROSAT observations, Chu et al. (2000) identified a compact source at the center of MCSNR J0507-6847 superposed on the star cluster HS122. This was proposed to be a BeXRB based on its coincidence with a star-cluster region, although no timing or spectral analysis could be performed to understand its nature. The position of the compact source proposed in Chu et al. (2000) is compatible with the now known quasar MQS J050736.44-684751.6, which overlaps with the SNR along the line of sight. The other point source in the vicinity, XMMU J050722.1-684758 has been identified as a BeXRB pulsar instead in this work which qualifies as the most probable compact object associated with MCSNR J0507-6847. It is likely that XMMU J050722.1-684758, owing to its intrinsic X-ray variability, was in a faint state during the ROSAT observations presented in Chu et al. (2000), and thus either undetected or confused with the quasar.

For the BeXRB to be associated to the SNR requires a corecollapse origin, with the parent SN producing both the remnant and leaving an NS behind. MCSNR J0507-6847 is in a star-forming region as evidenced from the presence of many upper main-sequence 


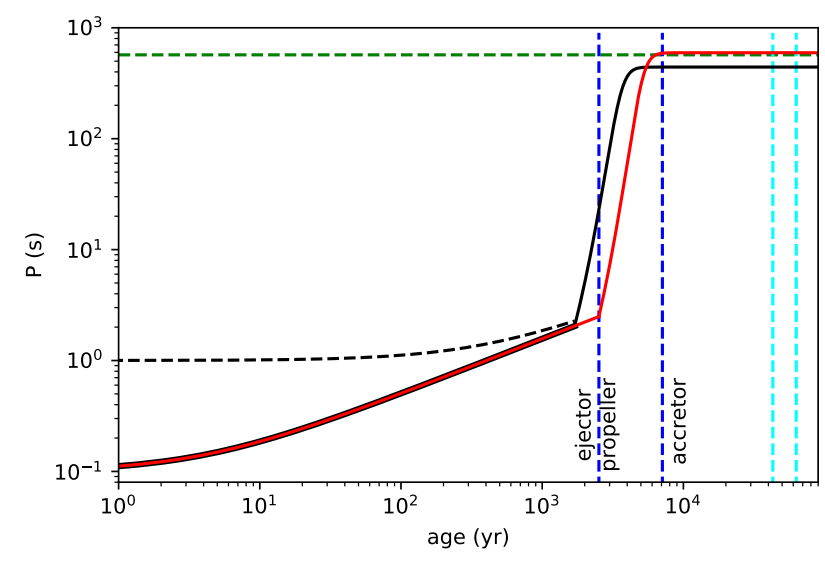

Figure 12. Spin evolution as a function of time marking the ejector, propeller and ejector phases of XMMU J050722.1-684758 following Ho et al. (2020). Assumed initial spin of the neutron star: $P_{0}$ is $100 \mathrm{~ms}$ (solid lines) and $1 \mathrm{~s}$ (dashed line). $B=4 \times 10^{14} \mathrm{G}$ and evolution is shown for a constant mass accretion rate of $\dot{M}=8 \times 10^{-12} M_{\odot} \mathrm{yr}^{-1}$ (red) and $2 \times 10^{-11} M_{\odot} \mathrm{yr}^{-1}$ (black). The green horizontal line marks the spin period and the cyan lines indicate the estimated age of the system, assuming the pulsar is indeed the progenitor of MCSNR J0507-6847.

stars in its surrounding, and confirmed by the reconstructed starformation history by Harris \& Zaritsky (2009). In a galaxy seen almost face-on like the LMC, the location of an SNR in such a star-forming region is a strong indication of a core-collapse origin, as opposed to galaxies with significant line-of-sight depth like the SMC where more confusion can arise. In the LMC, only one type Ia SNR can be clearly misidentified by looking solely at the star-forming environment of the object (Maggi et al. 2016). Coincidentally, this source is the nearby SNR N103B. Even in the absence of strong spectral evidence for a core-collapse SN, because the emission is dominated by LMC ISM, we thus conclude that MCSNR J0507-6847 was most likely created in a core-collapse SN.

Furthermore, the proximity of XMMU J050722.1-684758 near the geometrical centre of the SNR $\left(0.9^{\prime}\right)$, indicates the association of the BeXRB pulsar XMMU J050722.1-684758 with MCSNR J0507-6847. As the SNR has an almost perfect elliptical shape, the center of the ellipse is a good proxy for the likely explosion site and the origin of the NS in XMMU J050722.1-684758. This sets constraints on the NS transverse velocity. At the LMC distance of $50 \mathrm{kpc}$, the transverse velocity projected on the sky is:

$v_{\text {proj }}=284 \delta \theta\left(\frac{D}{50 \mathrm{kpc}}\right)\left(\frac{t_{\mathrm{SNR}}}{50 \mathrm{kyr}}\right)^{-1} \mathrm{~km} \mathrm{~s}^{-1}$

with $\delta \theta$ the angular separation in minutes of arc from the geometric centre of the SNR to the NS, and $t_{\mathrm{SNR}}$ the age of the SNR. Equating this to the dynamical age $t_{\text {dyn }}$ estimated for a Sedov model (Sect. 4.2, ranging from 43 to $63 \mathrm{kyr}$, and for $\delta \theta=0.9^{\prime}$, the projected velocity is 200 to $300 \mathrm{~km} \mathrm{~s}^{-1}$. The observed velocity distribution of radio pulsars indicate a mean pulsar transverse velocity of $345 \mathrm{~km} \mathrm{~s}^{-1}$, with an inferred three-dimensional velocity of $450 \mathrm{~km} \mathrm{~s}^{-1}$ (Lyne \& Lorimer 1994). The prediction for NSs in HMXBs is expected to be smaller and has diverse values in literature (on the order of 30$150 \mathrm{kms}^{-1}$; see Coe 2005; Bodaghee et al. 2012; Zuo et al. 2014; Zuo 2015). The inferred transverse velocity of the BeXRB pulsar XMMU J050722.1-684758 is slightly higher than expected from the current predictions and therefore further raises a question on its association with MCSNR J0507-6847. Moreover, it cannot be

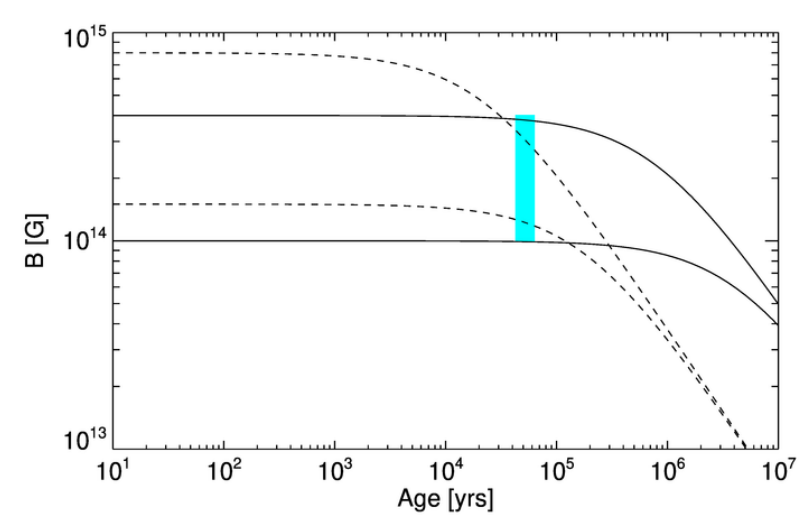

Figure 13. Magnetic field evolution assuming ambipolar diffusion in the irrotational mode (i.e. solid lines; $b=0.01$ and $\alpha=5 / 4$ ), or in the solenoidal mode (i.e. dashed lines; $b=0.15$ and $\alpha=5 / 4$ ), for different initial $B$ field strength. The loci of the calculated $B$ field assuming spin equilibrium for the age of the NS is marked with cyan color.

ruled out that even if the SNR and the NS originated from the same star cluster, the progenitors of both could be distinct.

\subsection{Constraints of NS properties at birth and the spin evolution of the system}

Evolutionary, NSs are thought to be born with short spin periods and strong magnetic fields $\left(P_{0} \sim 10-100 \mathrm{~ms}, B \sim 10^{13} \mathrm{G}\right.$; FaucherGiguère \& Kaspi 2006). At birth the BeXRB pulsar is thought to be at the ejector phase. In this regime, the pulsar does not interact with the accreting matter as the NS light cylinder is smaller than the magnetospheric radius as would be calculated by the balance of magnetic and gas pressure. As the NS spins down, at some point the magnetospheric radius and light cylinder become equal and the system transitions to the propeller phase, where the interactions of the disk and magnetosphere cause a faster spin down rate. Eventually an equilibrium is reached, however the whole evolution is complicated by a time variable accretion rate. The duration of each phase is a function of the NS $B$ field, and average mass accretion rate throughout the evolution. Following the prescription of Ho et al. (2020) we traced the evolutionary history of XMMU J050722.1-684758 given the estimate of the magnetic field obtained by equating the co-rotation and magnetospheric radius at spin-equilibrium:

$P_{\mathrm{eq}}=\left(\frac{4 \pi^{2} R_{\mathrm{M}}^{3}}{G M_{\mathrm{NS}}}\right)^{1 / 2}, R_{\mathrm{M}}=\frac{\xi}{2}\left(\frac{R_{\mathrm{NS}}^{12} B^{4}}{G M_{\mathrm{NS}} \dot{M}^{2}}\right)^{1 / 7}$

where $G$ is the gravitational constant, $M_{\mathrm{NS}}=1.4 \mathrm{M}_{\odot}$ and $R_{\mathrm{NS}}=$ $10 \mathrm{~km}$ is the NS mass and radius. This results into a magnetic field of $B \sim 4 \times 10^{14} \mathrm{G}$. Further, from the observed $L_{X}$ (i.e. $\sim 10^{35}$ $\mathrm{erg} \mathrm{s}^{-1}$ ) and by assuming that all dynamic energy of the in-falling material is converted to radiation we derive a mass accretion rate of $5 \times 10^{14} \mathrm{~g} \mathrm{~s}^{-1}$ (i.e. $L_{X} \approx 0.2 \dot{M} c^{2}$ ). Using eq. 7 and eq. 12 from Ho et al. (2020), Fig. 12 plots the complete evolution of the NS spin period from the ejector phase, to the propeller phase and finally to the accretor/spin equilibrium phase when the current measured spin period of the NS is achieved. From the measured $B$ and massaccretion rate the system crosses the propeller stage at $t \simeq 2600 \mathrm{yr}$ and reaches spin-equilibrium at $t \simeq 7000 \mathrm{yr}$. As is expected, the choice of the initial spin period $P_{0}$ does not affect the evolution of the system at later times, such as the accretor phase. We can obtain 
an upper limit on the birth spin of the NS by equating $R_{\mathrm{M}}$ with $R_{L C}$ (light-cylinder) and find $P_{0} \lesssim 2 \mathrm{~s}$.

The estimated magnetic field strength of $B \sim 4 \times 10^{14} \mathrm{G}$ is obtained by a simple prescription of equating the co-rotation and magnetospheric radius and assuming the scaling factor between magnetospheric radius $\left(R_{\mathrm{M}}\right)$ and Alfvén radius; $\xi \simeq 0.5$ (Campana et al. 2018). The $B$ here also refers to the field measured at the magnetic poles ( $\mu=B R^{3} / 2$, where $\mu$ is the magnetic dipole moment) and the field strength measured at the magnetic equator is lower by a factor of 2 . The results overall indicate a magnetar-like field strength of $B \gtrsim 10^{14} \mathrm{G}$.

The prescription of Ho et al. (2020) assumes a constant mass accretion rate and $B$ throughout the various stages undergone by the system. The $B$ on the other hand decays through non linear processes such as ambipolar diffusion and/or a Hall cascade (Goldreich \& Reisenegger 1992). Numerical simulations has shown that for $B>$ $10^{13} \mathrm{G}$ this evolution follows a simple decay law (Colpi et al. 2000), with the following form:

$B(t)=\frac{B_{0}}{\left[1+b \alpha B_{0}^{\alpha} t\right]^{1 / \alpha}}$,

where $B$ and $t$ quantities are in units of $10^{13} \mathrm{G}$ and $10^{6} \mathrm{yr}$ respectively, while values for parameters $b$ and $\alpha$ denote different modes of $B$ field decay (see Colpi et al. 2000, for details).

By following eq. 3 we can calculate the B field decay from its birth and compare it with its current value (see Fig 13). It is evident that minimal decay has occurred within this time and the pulsar has maintained its initial $B$ strength as is expected from the time scales expected from the magnetic field decay with is $\gtrsim 10^{6} \mathrm{yr}$.

\section{CONCLUSIONS}

The probable association of XMMU J050722.1-684758 with MCSNR J0507-6847 makes it the third identified BeXRB-SNR system. Pulsations are discovered at $570 \mathrm{~s}$ indicating the spin period of the neutron star. The estimated age of the SNR is 43-63 kyr which points to a middle aged to old SNR. The magnetic field strength of the neutron star is indicated to be $\gtrsim 10^{14} \mathrm{G}$. The other 2 such systems are SXP 1062 (Haberl et al. 2012; Hénault-Brunet et al. 2012) and SXP 1323 (Gvaramadze et al. 2019) both located in the Small Magellanic Cloud. This highlights the ideal environment and the suitability of finding such objects in the Magellanic Clouds. All of the three BeXRB-SNR systems (including our discovery) host slowly spinning pulsars and have similar estimates for the age of the parent SNR. The eROSITA instrument on board the Russian/German Spektrum-Roentgen-Gamma(SRG) mission all-sky survey will provide a deep and complete coverage of the Magellanic System for the first time in X-rays and will be instrumental in finding more such systems.

\section{ACKNOWLEDGEMENTS}

This work uses observations obtained with $X M M$-Newton, an ESA science mission with instruments and contributions directly funded by ESA Member States and NASA. The XMM-Newton project is supported by the DLR and the Max Planck Society. GV is supported by NASA Grant Number 80NSSC20K0803, in response to XMMNewton AO-18 Guest Observer Program. GV acknowledges support by NASA Grants number 80NSSC20K1107 and 80NSSC21K0213.
M.S. acknowledges support by the Deutsche Forschungsgemeinschaft through the Heisenberg professor grants SA 2131/5-1 and 12-1. The OGLE project has received funding from the National Science Centre, Poland, grant MAESTRO 2014/14/A/ST9/00121 to AU. This work is based on data from eROSITA, the primary instrument aboard SRG, a joint Russian-German science mission supported by the Russian Space Agency (Roskosmos), in the interests of the Russian Academy of Sciences represented by its Space Research Institute (IKI), and the Deutsches Zentrum für Luft- und Raumfahrt (DLR). The SRG spacecraft was built by Lavochkin Association (NPOL) and its subcontractors, and is operated by NPOL with support from the Max Planck Institute for Extraterrestrial Physics (MPE). The development and construction of the eROSITA X-ray instrument was led by MPE, with contributions from the Dr. Karl Remeis Observatory Bamberg \& ECAP (FAU Erlangen-Nürnberg), the University of Hamburg Observatory, the Leibniz Institute for Astrophysics Potsdam (AIP), and the Institute for Astronomy and Astrophysics of the University of Tübingen, with the support of DLR and the Max Planck Society. The Argelander Institute for Astronomy of the University of Bonn and the Ludwig Maximilians Universität Munich also participated in the science preparation for eROSITA. The eROSITA data shown here were processed using the eSASS/NRTA software system developed by the German eROSITA consortium. The Australian SKA Pathfinder is part of the Australia Telescope National Facility which is managed by CSIRO. Operation of ASKAP is funded by the Australian Government with support from the National Collaborative Research Infrastructure Strategy. ASKAP uses the resources of the Pawsey Supercomputing Centre. Establishment of ASKAP, the Murchison Radio-astronomy Observatory and the Pawsey Supercomputing Centre are initiatives of the Australian Government, with support from the Government of Western Australia and the Science and Industry Endowment Fund. We acknowledge the Wajarri Yamatji people as the traditional owners of the Observatory site.

\section{DATA AVAILABILITY}

X-ray data are available through the High Energy Astrophysics Science Archive Research Center heasarc.gsfc.nasa.gov. OGLE data are available through the OGLE XROM online portal http: //ogle.astrouw.edu.pl/ogle4/xrom/xrom.html.

\section{REFERENCES}

Ambrocio-Cruz P., Laval A., Marcelin M., Amram P., 1997, A\&A, 319, 973 Antoniou V., Zezas A., 2016, MNRAS, 459, 528

Arnaud K. A., 1996, in Jacoby G. H., Barnes J., eds, Astronomical Society of the Pacific Conference Series Vol. 101, Astronomical Data Analysis Software and Systems V. p. 17

Bodaghee A., Tomsick J. A., Rodriguez J., James J. B., 2012, ApJ, 744, 108 Bozzetto L. M., et al., 2017, ApJS, 230, 2

Campana S., Stella L., Mereghetti S., de Martino D., 2018, A\&A, 610, A46

Chu Y.-H., Kim S., Points S. D., Petre R., Snowden S. L., 2000, AJ, 119, 2242

Coe M. J., 2005, MNRAS, 358, 1379

Coe M. J., et al., 2012, MNRAS, 424, 282

Coe M. J., Bartlett E. S., Bird A. J., Haberl F., Kennea J. A., McBride V. A., Townsend L. J., Udalski A., 2015, MNRAS, 447, 2387

Colpi M., Geppert U., Page D., 2000, ApJ, 529, L29

Dickey J. M., Lockman F. J., 1990, ARA\&A, 28, 215

Dunne B. C., Points S. D., Chu Y.-H., 2001, ApJS, 136, 119

Evans P. A., et al., 2007, A\&A, 469, 379 
Faucher-Giguère C.-A., Kaspi V. M., 2006, ApJ, 643, 332

Fischer P., Welch D. L., Mateo M., 1993, AJ, 105, 938

Geldzahler B. J., Pauls T., Salter C. J., 1980, A\&A, 84, 237

Gilmozzi R., Kinney E. K., Ewald S. P., Panagia N., Romaniello M., 1994, ApJ, 435, L43

Goldreich P., Reisenegger A., 1992, ApJ, 395, 250

González-Galán A., Oskinova L. M., Popov S. B., Haberl F., Kühnel M., Gallagher J., Schurch M. P. E., Guerrero M. A., 2018, MNRAS, 475, 2809

Gregory P. C., Loredo T. J., 1996, ApJ, 473, 1059

Gvaramadze V. V., Kniazev A. Y., Oskinova L. M., 2019, MNRAS, 485, L6

Haberl F., Sturm R., 2016, A\&A, 586, A81

Haberl F., Sturm R., Filipović M. D., Pietsch W., Crawford E. J., 2012, A\&A, 537, L1

Haberl F., et al., 2017, A\&A, 598, A69

Haberl F., et al., 2020, The Astronomer's Telegram, 13609, 1

Harris J., Zaritsky D., 2009, AJ, 138, 1243

Heinz S., et al., 2013, ApJ, 779, 171

Hénault-Brunet V., et al., 2012, MNRAS, 420, L13

Ho W. C. G., Wijngaarden M. J. P., Andersson N., Tauris T. M., Haberl F., 2020, MNRAS, 494, 44

Kavanagh P. J., Sasaki M., Whelan E. T., Maggi P., Haberl F., Bozzetto L. M., Filipović M. D., Crawford E. J., 2015, A\&A, 579, A63

Kim S., Dopita M. A., Staveley-Smith L., Bessell M. S., 1999, AJ, 118, 2797

Kim S., Staveley-Smith L., Dopita M. A., Sault R. J., Freeman K. C., Lee Y., Chu Y.-H., 2003, ApJS, 148, 473

Kozłowski S., et al., 2013, ApJ, 775, 92

Kraft R. P., Burrows D. N., Nousek J. A., 1991, ApJ, 374, 344

Lomb N. R., 1976, Ap\&SS, 39, 447

Lyne A. G., Lorimer D. R., 1994, Nature, 369, 127

Maggi P., et al., 2012, A\&A, 546, A109

Maggi P., et al., 2016, A\&A, 585, A162

Maggi P., et al., 2019, A\&A, 631, A127

Maitra C., Paul B., Haberl F., Vasilopoulos G., 2018, MNRAS, 480, L136

Maitra C., et al., 2019, MNRAS, 490, 5494

Maitra C., et al., 2020, The Astronomer's Telegram, 13610, 1

Maitra C., Haberl F., Vasilopoulos G., Ducci L., Dennerl K., Carpano S., 2021, A\&A, in press

McLaughlin D. E., van der Marel R. P., 2005, ApJS, 161, 304

Predehl P., et al., 2020, arXiv e-prints, p. arXiv:2010.03477

Robertson J. W., 1974, A\&AS, 15, 261

Rosen S. R., et al., 2016, A\&A, 590, A1

Savitzky A., Golay M. J. E., 1964, Analytical Chemistry, 36, 1627

Scargle J. D., 1982, ApJ, 263, 835

Schenck A., Park S., Post S., 2016, AJ, 151, 161

Schmidtke P. C., Cowley A. P., Udalski A., 2013, MNRAS, 431, 252

Seward F. D., Charles P. A., Foster D. L., Dickel J. R., Romero P. S., Edwards Z. I., Perry M., Williams R. M., 2012, ApJ, 759, 123

Smith R. C., Points S., Aguilera C., Leiton R., Chu Y. H., Winkler P. F., MCELS 2004, in American Astronomical Society Meeting Abstracts. p. 101.08

Souchay J., Andrei A. H., Barache C., Bouquillon S., Suchet D., Taris F., Peralta R., 2012, A\&A, 537, A99

Strüder L., et al., 2001, A\&A, 365, L18

Sturm R., et al., 2013, A\&A, 558, A3

Turner M. J. L., et al., 2001, A\&A, 365, L27

Udalski A., Szymanski M., Kaluzny J., Kubiak M., Mateo M., 1992, Acta Astron., 42, 253

Udalski A., Szymański M. K., Szymański G., 2015, Acta Astron., 65, 1

Vallenari A., Aparicio A., Fagotto F., Chiosi C., Ortolani S., Meylan G., 1994, A\&A, 284, 447

Vasilopoulos G., Maggi P., Haberl F., Sturm R., Pietsch W., Bartlett E. S., Coe M. J., 2013, A\&A, 558, A74

Vasilopoulos G., Haberl F., Sturm R., Maggi P., Udalski A., 2014, A\&A, 567, A129

Vasilopoulos G., Maitra C., Haberl F., Hatzidimitriou D., Petropoulou M., 2018, MNRAS, 475, 220
Verner D. A., Ferland G. J., Korista K. T., Yakovlev D. G., 1996, ApJ, 465, 487

Watson M. G., et al., 2009, A\&A, 493, 339

Wilms J., Allen A., McCray R., 2000, ApJ, 542, 914

Yew M., et al., 2020, MNRAS, 500, 2336

Zuo Z.-Y., 2015, A\&A, 573, A58

Zuo Z.-Y., Li X.-D., Gu Q.-S., 2014, MNRAS, 437, 1187

van Jaarsveld N., Buckley D. A. H., McBride V. A., Haberl F., Vasilopoulos G., Maitra C., Udalski A., Miszalski B., 2018, MNRAS, 475, 3253

van der Heyden K. J., Bleeker J. A. M., Kaastra J. S., 2004, A\&A, 421, 1031

This paper has been typeset from a $\mathrm{T}_{\mathrm{E}} \mathrm{X} / \mathrm{LT}_{\mathrm{E}} \mathrm{X}$ file prepared by the author. 